\title{
Evaluation of the Protection against Infectious Bursal Disease (IBD) Challenge in Progeny Born to Parents Having Received a Vaccination Program Using a Herpesvirus of Turkey-Infectious Bursal Disease (HVT-IBD) Vector Vaccine
}

\author{
Stephane Lemiere ${ }^{1}$, Jean-Claude Gauthier ${ }^{2}$, Angeli Kodjo ${ }^{3}$, Laure Vinit ${ }^{3}$, Andrea Delvecchio ${ }^{1}$, \\ Francesco Prandini ${ }^{1}$ \\ ${ }^{1}$ Merial S.A.S., Lyon, France; ${ }^{2}$ Lycee D’Enseignement General Agricole de Vendome, Vendome, France; ${ }^{3}$ VetAgro Sup, Marcy \\ L'Etoile, France. \\ Email: stephane.lemiere@merial.com
}

Received February $21^{\text {st }}, 2013$; revised March $25^{\text {th }}, 2013$; accepted April $3^{\text {rd }}, 2013$

Copyright (C) 2013 Stephane Lemiere et al. This is an open access article distributed under the Creative Commons Attribution License, which permits unrestricted use, distribution, and reproduction in any medium, provided the original work is properly cited.

\begin{abstract}
Broiler breeder vaccination against IBD is usually based on the injection of at least one inactivated vaccine in oil adjuvant, typically included in a combined vaccine. Priming using one or several IBD vaccine (s) has been the most common way to immunize the breeders so far. In summary, protection against vvIBD challenge in chicks of one commercial genetic line vaccinated in ovo with the HVT-IBD vector vaccine was demonstrated. The parents' IBD vaccination program, using the HVT-IBD vector vaccine alone, the HVT-IBD vector vaccine plus IBD inactivated vaccine, and inactivated IBD vaccine alone, did not impair their progeny's in ovo HVT-IBD vector vaccine take and subsequent protection against vvIBD virus challenge. An advantage in terms of immunization of the progeny against vvIBD was shown in the chicks born to breeders vaccinated with the HVT-IBD vaccine as a primer, as compared to breeders vaccinated with the inactivated vaccine alone. High level of IBD maternally-derived antibodies transmitted to the progeny by their parents induces together with an early onset of immunity by in ovo injection of a HVT-IBD vector vaccine clinical protection, as monitored on bursas, after vvIBD virus challenge.
\end{abstract}

Keywords: Infectious Bursal Disease Virus; Vector Vaccine; ELISA Serology; Protection; Progeny

\section{Introduction}

Infectious Bursal Disease is a viral disease of chickens that can be controlled by vaccination [1]. Vaccination strategy is dual: passive protection induced by the maternally-derived antibodies (MDA) and active immunization of the progeny by its vaccination [2,3], taking into account the interference between these MDAs and the live vaccine take. A very virulent form of the disease was described around twenty years ago $[4,5]$ and is still considered as a major treat to the poultry industry everywhere in the world [6], and more recently in the United States of America [7]. Very virulent (vv) IBD infections lead to severe clinical signs of IBD and high mortality. The clinical signs are typical of those an acute immunodepression, with depression, prostration of the affected birds, diarrhoea, during the first weeks of life [6]. IBD leads to bursa lesions and immunodepression [8]. Broiler breeder vaccination against IBD is usually based on the injection of at least one inactivated vaccine in oil adjuvant [9], typically included in a combined vaccine. Priming using one or several live IBD vaccine (s) has been the most common way to immunize the breeders so far, as early protection against IBD is required by vaccination programs of the breeders. The use of a HVT-IBD vector vaccine injected at day-old to future broiler breeders $[10,11]$ has been investigated since the launch of this type of vaccine intended for day-old vaccination of chickens. A vaccination against Marek's Disease (MD) in future breeders requires a Rispens serotype 1 vaccine to be mixed with the HVT-IBD vector vaccine [12]. The 
objective of the studies of protection against vvIBD challenge of progeny born to breeders vaccinated using different vaccination programs based on HVT-IBD vector vaccine, and vaccinated with the HVT-IBD vector vaccine in ovo, was to validate the proper vaccination program (s) of the breeders. Additionally, the kinetics of decrease of maternally derived IBD antibodies detected by two commercial ELISA kits [13] in progeny born to the same breeders was studied.

\section{Material and Methods}

\subsection{Material}

\subsubsection{Vaccines}

The vaccine strain is a vector HVT vaccine virus, strain FC126. Its genome contains the VP2 (Viral protein 2) gene of the 52/70 Faragher strain of IBDV [14]. It is the vHVT013-69 strain registered as VAXXITEK ${ }^{\circledR}$ HVT + IBD (Merial S.A.S., Lyon, France) (VAXXITEK is a registered trademark of Merial in the United States of America and elsewhere). Vaccine from a commercially available HVT-IBD vector vaccine batch in France was used to vaccinate the future breeders manually at one day of age for the laboratory studies. It was diluted into a diluent intended to HVT-IBD vector cell-associated vaccine preparation together with a commercial Rispens serotype 1 Marek's Disease vaccine. Individual dosage was a volume of $0.2 \mathrm{~mL}$ per day-old chick, according to the Manufacturer's instructions. Progeny was itself vaccinated with the HVT-IBD vector vaccine in ovo at 18 days of embryonation with a classical automatic in ovo injector (AviTech, LLC., Salisbury, MD, United States of America). A commercial inactivated in oil adjuvant IBD monovalent vaccine registered in France (Merial S.A.S., Lyon, France), VNJO strain, was injected prior to the laying period, at 16 weeks of age. Dosage was 0.3 $\mathrm{mL}$ for intramuscular injection.

\subsubsection{Chickens}

Day-old commercial future breeders were included in the studies. They were COBB 500 breeders (Cobb-Vantress, Putten, the Netherlands) (COBB is a registered trademark of Cobb-Vantress in the United States of America and elsewhere) placed in conventional facilities with controlled access in France in the context of an experimental farm (Lycée d'enseignement général et technologique agricole, Vendôme, France). Three groups of about ten breeder hens with one male were reared contemporaneously. Eggs were collected daily throughout the laying period and incubated at the farm. Chicks were born to these conventional breeders and shipped to a confined A2 facility for rearing prior to challenge test (VetAgro Sup Institut Claude Bourgelat, Marcy L'Etoile, France).

\subsubsection{Challenge Virus}

Challenge using vvIBD virus was performed with a volume of $0.1 \mathrm{~mL}$ of the strain $89163 / 7.3$ (ANSES, Plou fragan, France) at the titre of $4.0 \log 10$ Egg Infectious Dose $50 \%\left(\mathrm{EID}_{50}\right)$, at 24 days of age (D24), when IBD antibodies waned. All birds were challenged, using the intra-nasal route. Challenge validation was performed using a classic ELISA kit detecting antibodies against full IBD viruses (Synbiotics, Corp., Kansas City, MO, United States of America).

\subsubsection{Serology}

Sera were tested for vaccine take monitoring, as well as decrease of MDA using two ELISA commercial kits (Synbiotics, Corp., Kansas City, MO, United States of America), the ProFLOK ${ }^{\circledR}$ Ab IBD Plus ELISA and the classic ProFLOK Ab IBD ELISA (ProFLOK is a registered trademark of Synbiotics in the United States of America and elsewhere). The first test is considered as more sensitive as the antigen is a purified extract of IBD virus infected bursa tissue [13]. It allows early detection, during the observation period of the chickens within the study, of the post-HVT-IBD vector vaccine vaccination antibody detection, whereas the second kit only allows detection of full IBD virus antibody detection [13].

\subsubsection{Vaccination Procedure}

Two experiments, trials I \& II, were performed in progeny born to three groups of breeders vaccinated with either the HVT-IBD vector vaccine alone at day-old, or the HVT-IBD vector vaccine as a primer of a vaccination using the inactivated IBD vaccine, or the inactivated IBD vaccine alone.

\subsection{Methods}

\subsubsection{Experiment I (vvIBD Protection of Progeny Studies)}

Breeders were vaccinated with three different combinations of IBD vaccines based on HVT-IBD vector vaccine and monovalent inactivated IBD vaccine (GC for Groups Challenge) (Table 1).

Progeny was vaccinated in ovo with the HVT-IBD vector vaccine Parameters of study of vvIBD challenge were progeny bursa to bodyweight $(\mathrm{b} / \mathrm{bw})$ ratio and histological bursa lesion scoring $[11,15,16]$ at day of challenge, D24, D24, then at D28, D31 and D34 of age, following the vvIBD challenge. $\mathrm{b} / \mathrm{bw}$ ratios were expressed by dividing the bursa weight expressed in grams by the body weight expressed in grams as well, the whole multiplied by 1000 in order to facilitate reading the results. Results below 1 can be considered as pathologic [13]. Bursa histological score was set using a scale between 1 and 4 [16]. Results above 2 can be considered as pathologic [16]. 
Table 1. Experiment I. vvIBD challenge study group repartition.

\begin{tabular}{|c|c|c|c|}
\hline Groups & $\begin{array}{c}\text { Breeder IBD vaccination } \\
\text { program }\end{array}$ & $\begin{array}{l}\text { vvIBD challenge } \\
\text { at D24 of age }\end{array}$ & $\begin{array}{r}\text { Number o } \\
\text { chickens }\end{array}$ \\
\hline $\mathrm{GC} 1 \mathrm{c}$ & HVT-IBD vector vaccine & + & 8 \\
\hline $\mathrm{GC} 2 \mathrm{c}$ & $\begin{array}{l}\text { HVT-IBD vector vaccine }+ \\
\text { inactivated IBD vaccine }\end{array}$ & + & 8 \\
\hline $\mathrm{GC} 3 \mathrm{c}$ & Inactivated IBD vaccine & + & 8 \\
\hline GC1u & HVT-IBD vector vaccine & - & 8 \\
\hline $\mathrm{GC} 2 \mathrm{u}$ & $\begin{array}{l}\text { HVT-IBD vector vaccine }+ \\
\text { inactivated IBD vaccine }\end{array}$ & - & 8 \\
\hline $\mathrm{GC} 3 \mathrm{u}$ & Inactivated IBD vaccine & - & 8 \\
\hline
\end{tabular}

Serological monitoring using the classic IBD ELISA kit was performed in all the birds on day of challenge, D24, then on D28, D31 and D34. Each population of progeny was split into two groups, one challenged with the vvIBD virus, and the other kept unchallenged, as a control $(\mathrm{GC} 1 \mathrm{c}=$ HVT-IBD vector vaccine alone, challenged progeny; $\mathrm{GClu}=$ HVT-IBD vector vaccine alone, unchallenged progeny; GC2c $=$ HVT-IBD vector vaccine plus inactivated IBD vaccine, challenged progeny; $\mathrm{GC} 2 \mathrm{u}$ $=$ HVT-IBD vector vaccine plus inactivated IBD vaccine, unchallenged progeny; $\mathrm{GC} 3 \mathrm{c}=$ inactivated IBD vaccine, challenged progeny; $\mathrm{GC} 3 \mathrm{u}=$ inactivated $\mathrm{IBD}$ vaccine, unchallenged progeny). Both challenged and unchallenged chickens were in separate rooms. Both rooms' ventilation and personnel circulation were tightly secured and no challenge vvIBD virus could enter the unchallenged chicken room. Classic IBD ELISA was performed in order to validate contact or absence of contact with the vvIBD challenge virus. Protection against vvIBD challenge was assessed by b/bw ratio and bursa lesion scoring in controls and vaccinates for each breeder vaccination program. Comparison between the breeders' vaccination programs was established, HVT-IBD vector vaccine alone, HVT-IBD vector vaccine with inactivated IBD vaccine booster, and inactivated IBD vaccine alone. Serological response to vvIBD challenge was one of ways to monitor the challenge model.

\subsubsection{Experiment II (Maternally-Derived IBD Antibody Decrease Studies)}

For study of IBD passive immunity conventional chickens for their immune status and born to the three groups of breeders (GS for Groups Serology) (GS1 = HVT-IBD vector vaccine alone; GS2 $=$ HVT-IBD vector vaccine plus inactivated IBD vaccine; GS3 = inactivated IBD vaccine) were kept unvaccinated (Table 2). These chickens were born to the same breeders as the previous study of protection against vvIBD challenge. They were hatched and then blood sampled individually at day old,
Table 2. Experiment II. IBD ELISA decrease study group repartition.

\begin{tabular}{|c|c|c|c|c|c|}
\hline Groups & $\begin{array}{l}\text { Breeder IBD } \\
\text { vaccination } \\
\text { program }\end{array}$ & $\begin{array}{c}\text { Number } \\
\text { of } \\
\text { chickens } \\
\text { at D1 }\end{array}$ & $\begin{array}{c}\text { Number } \\
\text { of } \\
\text { chickens } \\
\text { at D12 }\end{array}$ & $\begin{array}{c}\text { Number } \\
\text { of } \\
\text { chickens } \\
\text { at D21 }\end{array}$ & $\begin{array}{c}\text { Number } \\
\text { of } \\
\text { chickens } \\
\text { at D34 }\end{array}$ \\
\hline GS1 & $\begin{array}{c}\text { HVT-IBD } \\
\text { vector vaccine }\end{array}$ & $16^{*}$ & 16 & 16 & 16 \\
\hline GS2 & $\begin{array}{c}\text { HVT-IBD } \\
\text { vector vaccine } \\
+ \text { inactivated } \\
\text { IBD vaccine }\end{array}$ & $16^{*}$ & 16 & 16 & 15 \\
\hline GS3 & $\begin{array}{l}\text { Inactivated } \\
\text { IBD vaccine }\end{array}$ & $16^{*}$ & 16 & 16 & 16 \\
\hline
\end{tabular}

GS = Groups for Serology; ${ }^{*}$ blood sampling after humane euthanasia.

D12, D21 and D34. All serum samples were processed using the IBD Plus commercial ELISA, from day old up to D30 of age (Table 2).

\subsubsection{Statistical Analysis}

Challenge and unchallenged group results were compared for the $\mathrm{b} / \mathrm{bw}$ ratio, bursa scores and the IBD Plus ELISA titres. Non parametric Mann \& Whitney test for pair-wise comparisons was used for statistical analysis after prior evaluation of distribution of the variables to study. The signification level was set at $\mathrm{p} \leq 0.05$. All calculations were performed using SYSTAT (Crane Software International, Itd, Bangalore, India) software, version 12.0, for WINDOWS.

\section{Results}

\subsection{Experiment I}

Bursa to bodyweight ratio monitoring, as well as bursa lesion score monitoring, was established throughout the study at D28, D31 and D34. A significant difference in mean $\mathrm{b} / \mathrm{bw}$ ratio was noticed between groups $\mathrm{GC} 3 \mathrm{c}$ and $\mathrm{GC} 3 \mathrm{u}$ at D31, seven days post-challenge $(\mathrm{p}=0.012)$. Incomplete protection against vvIBD challenge measured by $b / b w$ ratio occurred at one time point (7 days postchallenge) in the progeny born to IBD inactivated vaccine alone vaccinates. No other significant difference between challenged and unchallenged groups, regardless the breeders' vaccination program, was noticed (Figure 1). The broiler chickens were protected against vvIBD according to the $\mathrm{b} / \mathrm{bw}$ ratio if they were born to breeders vaccinated at least with one shot of HVT-IBD vaccine. A significant difference in mean bursa histological score was noticed between groups $\mathrm{GC} 3 \mathrm{c}$ and $\mathrm{GC} 3 \mathrm{u}$ at $\mathrm{D} 28$, four days post-challenge $(p=0.007)$ and at D31, seven days post-challenge $(p=0.018)$. Incomplete protection against vvIBD challenge measured by bursa lesion histological score occurred at two time points (4 and 7 days post-challenge) in the progeny born to IBD inactivated 
vaccine alone vaccinates. No other significant difference between challenged and unchallenged groups, whatever the vaccination program of the breeders was noticed. The broiler chickens were protected against vvIBD according to the bursa histological lesion score if they were born to breeders vaccinated at least with one shot of HVT-IBD vaccine (Figure 2). IBD classic ELISA antibody production was monitored after vvIBD virus challenge test during 10 days. A significant difference in ELISA IBD classic mean titres was noticed between groups $\mathrm{GC} 3 \mathrm{c}$ and GC3u at two time points, D31 ( $p=0.004)$ and D34 $(p=$ 0.008 ) (Figure 3), displaying early post-challenge seroconversion.

\subsection{Experiment II}

IBD Plus ELISA maternally-derived antibody decrease kinetics was established. A significant difference in IBD Plus ELISA mean antibody titres was noticed between groups GS1 and GS3 at all time points, D1, D12, D21 and D34 of age (at D1, p = 0.036; at D12, D21, D34, p < 0.001 ), and between groups GS2 and GS3 at D12, D21, D34 ( $<<0.001)$. No significant difference within the same IBD ELISA kit mean titres was noticed between groups GS1 and GS2, progeny born to breeders having received at least a shot of HVT-IBD vaccine at day-old (Figure 4).

\section{Discussion}

Validation of the challenge model was evidenced by the reported significant differences between vvIBD virus challenged and unchallenged in one group of study, the GC3, at 7 and 10 days post-challenge, and by the fact that all the unchallenged groups of chickens showing classic IBD ELISA mean titres below 500 did not experience any vvIBD virus circulation, the titre being

b/bw ratio monitoring

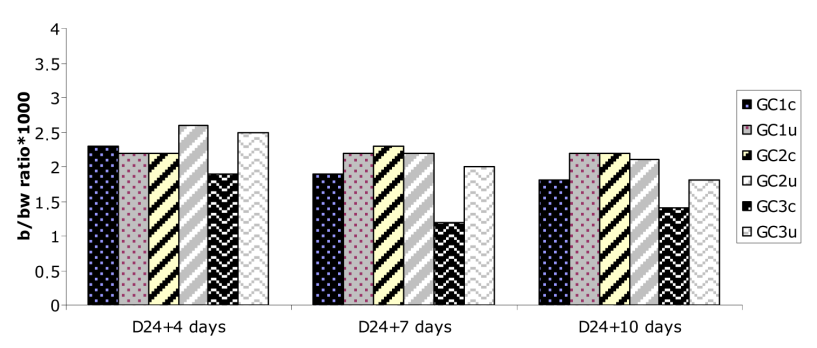

Figure 1. Bursa to bodyweight (b/bw) ratio monitoring further to vvIBD challenge at D24 of age ("VvIBD" into brackets in the legend means "challenged by vvIBD virus"). GC = Groups for Challenge; $c$ = challenged; $u$ = unchallenged. *Significant difference between groups GC3c and GC3u at D24 +7 days, seven days post-challenge $(p=0.012)$. The broiler chickens were protected against VvIBD according to the $b / b w$ ratio if they were born to breeders vaccinated at least with one shot of HVT-IBD vaccine.
Bursa histological lesion monitoring

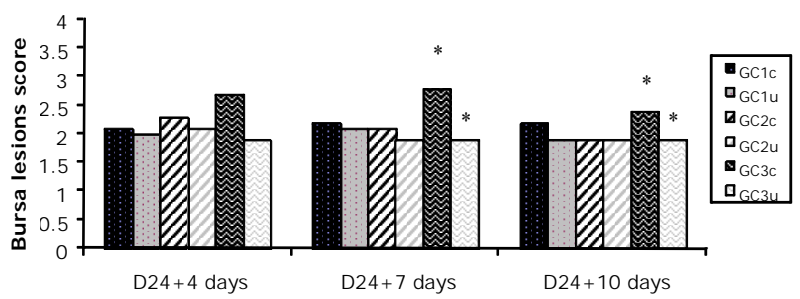

Figure 2. Bursa lesion histological score monitoring further to vvIBD challenge at D24 of age ("VvIBD" into brackets in the legend means "challenged by vvIBD virus"). GC = Groups for Challenge; $\mathbf{c}=$ challenged; $\mathbf{u}=$ unchallenged. "Significant difference between groups GC3c and GC3u at D24 +4 days, four days post-challenge $(p=0.007)$ and at D24 + 7 days, seven days post-challenge $(p=0.018)$. The broiler chickens were protected against vvIBD according to the bursa histological lesion score if they were born to breeders vaccinated at least with one shot of HVT-IBD vaccine.

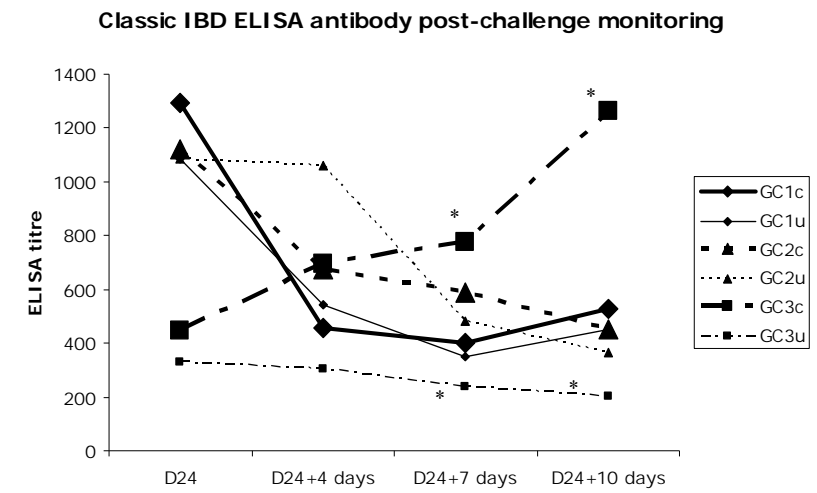

Figure 3. Post-vvIBD virus challenge classic IBD ELISA monitoring. GC = Groups for Challenge; $c$ = challenged; $u$ $=$ unchallenged. "Significant difference between groups GC3c and GC3u at D24 + 7 days, seven days post-challenge $(p=0.004)$, at $\mathrm{D} 24+10$ days, ten days post-challenge $(p=$ 0.008). Early post-challenge seroconversion was observed.

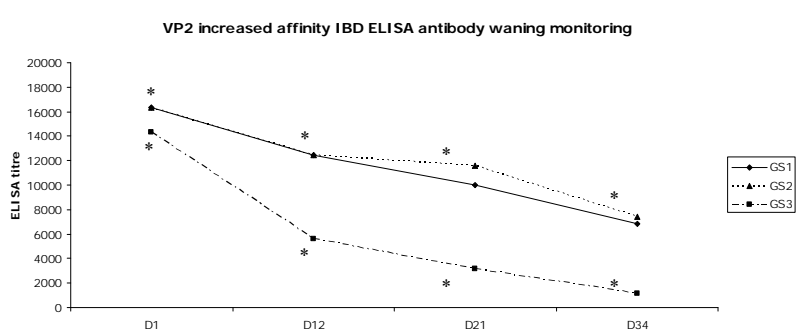

Figure 4. Maternally-derived IBD Plus ELISA antibody waning monitoring. GS = Groups for Serology. *Significant difference between groups GS1 and GS3 at all time points, D1, D12, D21 and D34 of age (at D1, p = 0.036; at D12, D21, D34, p $<0.001$ ), and between groups GS2 and GS3 at D12, D21, D34 $(p<0.001)$. No significant difference within the same IBD ELISA kit mean titres was noticed between groups GS1 and GS2, progeny born to breeders having received at least a shot of HVT-IBD vaccine at day-old. 
compatible with remaining MDAs [16]. All the vvIBD challenged chickens were reared in different pens within the same room and on litter in order to mimic the field contamination. That allowed contact between all the groups shedding the vvIBD challenge virus. As already mentioned, IBD classic ELISA results in the unchallenged birds evidenced that there had been no contact with the vvIBD virus present in the other room within the same A2 zone of the Institut Claude Bourgelat facilities.

Transmission of IBD maternal passive immunity is considered as an efficacious mechanism in the chicken species [17] and was demonstrated by the means of the IBD plus ELISA antibody monitoring in unvaccinated chickens in this study. Protection against vvIBD challenge was set at around 3000 in titre [13]. Passive protection could be estimated as complete throughout the observation period in chickens born to breeders having been vaccinated at least with one shot of the HVT-IBD vector vaccine at day-old, when that level of antibody protection was significantly inferior in the chickens born to breeders having been vaccinated with the IBD inactivated vaccine alone. Using this criterion, the interest of vaccinating breeders with the HVT-IBD vector vaccine finds its justification.

Onset of immunity against IBD induced by in ovo vaccination of the progeny was demonstrated by high titres of IBD plus ELISA antibodies, above 10,000, the day of vvIBD challenge in all the chickens born to breeders vaccinated with at least one HVT-IBD vector vaccine. Even the other chickens born to breeders vaccinated with the inactivated IBD vaccine alone showed lower titres, but above the threshold up to D21, at the time of full protection induced by active immunity of the HVT-IBD vector vaccine [11] administered at day old. No immunity gap occurred as the titres were up in the unchallenged chickens, whatever their immune status at birth. Inclusion of an IBD inactivated vaccine breeder group into the study was decided in order to evaluate the role of this compound in the vaccination program in terms of protection against vvIBD challenge and serology of progeny.

Importance of the breeders' vaccination program for clinical protection of progeny against vvIBD challenge was the main finding. It seems that a high level of MDA transmitted to the progeny induces together with an early onset of immunity by in ovo injection of a HVT-IBD vector vaccine provides full protection against the deleterious effects of a possible vvIBD virus circulation, as it can occur in field conditions. Referring to the classic IBD ELISA antibody production post-vvIBD virus challenge, it appears that the combination of high titres of IBD Plus ELISA MDA (Figure 4) and of the early onset of immunity induced by the in ovo injection of the HVT-IBD vector vaccine [10] in presence of MDA induces a clinical protection against vvIBD at D24 of age with no seroconversion against the vvIBD challenge virus, as shown by the results of the classic post-challenge IBD ELISA results. This seroconversion is observed only in the group of progeny born to IBD inactivated vaccine alone vaccinated breeders that displayed less protection against vvIBD monitored by $\mathrm{b} / \mathrm{bw}$ ratio, bursa scoring and passive immunity against IBD; it was stated already that it was related to post-vvIBD virus challenge seroconversion. It is clear that a breeder's vaccination program using at least one HVT-IBD vector vaccine applied at day-old could be the base for an optimal vaccination program. In any case, as breeders are chickens, they may be protected against vvIBD challenge by this single vaccination usually performed with a full MD vaccination program [12]. Nevertheless, in order to secure diversity of antibody transmission to progeny [17], it is probably preferable to add a re-vaccination with an inactivated full virus vaccine usually included into any broiler breeder vaccination program.

\section{Acknowledgements}

The authors want to give special thanks to Sylvie Gauthier for breeder rearing in the context of the experimental farm of Vendome, France.

\section{REFERENCES}

[1] H. E. Müller, E. Mundt, N. Eterradossi and M. R. Islam, "Current Status of Vaccines against Infectious Bursal Disease," Avian Pathology, Vol. 41, No. 2, 2012, pp. 133139. doi:10.1080/03079457.2012.661403

[2] H. K. Block, K. Meyer-Block, D. E. Rebeski, H. Scharr, S. de Wit, K. Rohn and S. Rautenschlein, "A Field Study on the Significance of Vaccination against Infectious Bursal Disease Virus (IBDV) at the Optimal Time Point in Broiler Flocks with Maternally Derived IBDV Antibodies," Avian Pathology, Vol. 36, No. 5, 2007, pp. 401-409. doi: $10.1080 / 03079450701589175$

[3] T. P. van den Berg and G. Meulemans, "Acute Infectious Bursal Disease in Poultry; Protection Afforded by Maternally Derived Antibodies and Interference with Live Vaccination," Avian Pathology, Vol. 20, No. 3, 1991, pp. 409-421. doi:10.1080/03079459108418779

[4] N. J. Chettle, J. C. Stuart and P. J. Wyeth, "Outbreak of Virulent Infectious Bursal Disease in East Anglia," Veterinary Record, Vol. 125, No. 10, 1989, pp. 271-272. doi:10.1136/vr.125.10.271

[5] T. P. van den Berg, M. Gonze and G. Meulemans, "Acute Infectious Bursal Disease in Poultry: Isolation and Characterisation of a Highly Virulent Strain," Avian Pathology, Vol. 20, No. 1, 1991, pp. 133-143. doi:10.1080/03079459108418748

[6] T. P. van den Berg, "Acute Infectious Bursal Disease in Poultry: A Review," Avian Pathology, Vol. 29, No. 3, 
2000, pp. 175-194. doi:10.1080/03079450050045431

[7] D. J. Jackwood, S. E. Sommer-Wagner, A. S. Stoute, P. R. Woolcock, B. M. Crossley, S. K. Hietala and B. R. Charlton, "Characteristics of a Very Virulent Infectious Bursal Disease from California," Avian Diseases, Vol. 53, No. 4, 2009, pp. 592-600. doi:10.1637/8957-061109-Reg.1

[8] J. C. Muskett, I. G. Hopkins, K. R. Edwards and D. H. Thornton, "Comparison of Two Infectious Bursal Disease Vaccine Strains: Efficacy and Potential Hazards in Susceptible and Maternally Immune Birds," Veterinary Record, Vol. 104, No. 15, 1979, pp. 332-334. doi:10.1136/vr.104.15.332

[9] R. A. Maas, S. Venema, H. L. Oei, J. M. A. Pol, I. J. T. M. Claassen and A. A. H. T. M. ter Huurne, "Efficacy of Inactivated Infectious Bursal Disease (IBD) Vaccines: Comparison of Serology with Protection of Progeny Chickens against IBD Virus Strains of Varying Virulence," Avian Pathology, Vol. 30, No. 4, 2001, pp. 345354. doi:10.1080/03079450120066359

[10] M. Bublot, N. Pritchard, F. X. Le-Gros and S. Goutebroze, "Use of a Vectored Vaccine against Infectious Bursal Disease of Chickens in the Face of High-Titred Maternally Derived Antibody," Journal of Comparative Pathology, Vol. 137, Sup 1, 2007, pp. S81-S84.

[11] S. Goutebroze, M. Curet, M. L. Jay, C. Roux and F. X. Le-Gros, "Efficacy of a Recombinant Vaccine HVT-VP2 against Gumboro Disease in the Presence of Maternal Antibodies," British Poultry Science, Vol. 44, No. 5, 2003, pp. 824-825. doi:10.1080/00071660410001667051

[12] S. Lemiere, S. Y. Wong, A. L. Saint-Gerand, S. Goutebroze and F. X. Le-Gros, "Compatibility of Turkey Herpesvirus-Infectious Bursal Disease Vector Vaccine with Marek's Disease Rispens Vaccine Injected into Day-Old
Pullets," Avian Diseases, Vol. 55, No. 1, 2011, pp. 113118. doi:10.1637/9507-081810-ResNote.1

[13] F. X. Le-Gros, A. Dancer, C. Giacomini, L. Pizzoni, M. Bublot, M. Graziani and F. Prandini, "Field Efficacy Trial of a Novel HVT-IBD Vector Vaccine for 1-Day-Old Broilers," Vaccine, Vol. 27, No. 4, 2009, pp. 592-596. doi:10.1016/j.vaccine.2008.10.094

[14] R. Darteil, M. Bublot, E. Laplace, J. F. Bouquet, J. C. Audonnet and M. Riviere, "Herpesvirus of Turkey Recombinant Viruses Expressing Infectious Bursal Disease Virus (IBDV) VP2 Immunogen Induce Protection against an IBDV Virulent Challenge in Chickens," Virology, Vol. 211, No. 2, 1995, pp. 481-490. doi:10.1006/viro.1995.1430

[15] J. M. Sharma, J. E. Dohmas and A. L. Metz, "Comparative Pathogenesis of Serotype 1 Isolates of Infectious Bursal Disease Virus and Their Effect on Humoral and Cellular Immune Competence of Specific-Pathogen-Free Chickens," Avian Diseases, Vol. 33, No. 1, 1989, pp. 112-124. doi:10.2307/1591076

[16] W. Solano, J. J. Giambrone and V. S. Panangala, "Comparison of a Kinetic-Based Enzyme-Linked Immunosorbent Assay (KELISA) and Virus-Neutralization Test for Infectious Bursal Disease Virus. II. Decay of Maternal Antibody in Progeny from White Leghorns Receiving Various No. 1, s Vaccination Regimens," Avian Diseases, Vol. 30, No. 1, 1986, pp. 126-131. doi:10.2307/1590623

[17] A. S. Abdel-Moneim and M. M. A. Abdel-Gawad, "Genetic Variations in Maternal Transfer and Immune Responsiveness to Infectious Bursal Disease Virus," Veterinary Microbiology, Vol. 114, No. 1-2, 2006, pp. 16-24. doi:10.1016/j.vetmic.2005.10.037 